\title{
Les recherches d'aujourd'hui sont les pratiques de demain : promouvoir une culture de la recherche
}

\author{
par Marc M. Perreault
}

Si je prends un peu de recul pour comparer la pratique de la $\checkmark$ pharmacie d'aujourd'hui à celle d'il y a dix ans, tous contextes confondus, est-ce que j'observe une différence? Pas suffisamment, et ce, malgré le fait que la plupart des provinces accordent maintenant aux pharmaciens le droit d'exercer de nouvelles activités, le tout dans le but d'améliorer les soins aux patients. Légalement, nous possédons maintenant de nouveaux outils pour optimiser la pharmacothérapie des patients : qu'il s'agisse de renouveler des ordonnances pour les maladies chroniques ou de prescrire des examens de laboratoire qui permettent la surveillance et le suivi de la pharmacothérapie ou de traiter des affections mineures. Individuellement et collectivement, nous nous consacrons tous au bien-être de nos patients en essayant d'obtenir des résultats optimaux à l'aide de services centrés sur le patient qui font appel à nos connaissances et grâce à une collaboration interprofessionnelle, peu importe notre milieu de pratique ${ }^{1}$. Or, une bonne part du potentiel qu'offrent ces occasions et buts demeure inexploitée.

Même si les pharmaciens n'ont pas encore adopté l'ensemble du champ de pratique clinique à leur disposition pour gérer plus efficacement la pharmacothérapie des patients, les avancées futures de services centrés sur le patient et fondés sur des données probantes dépendront de nos capacités en recherche. Ainsi, si nous souhaitons croître en tant que profession et vraiment nous efforcer de prodiguer les meilleurs soins, nous avons besoin non seulement de plus de recherches fondées sur la pratique, mais en outre de recherches plus solides ${ }^{2}$. Bien que d'excellentes pratiques soient nées de recherches menées au Canada et ailleurs ${ }^{3,4}$, ces types de pratique ne sont toujours pas largement adoptés et aucun objectif de recherche n'a été décrété pour la pratique de la pharmacie. J'invite fortement notre profession à aller de l'avant et à adopter les pratiques dont les avantages ont déjà été démontrés et je l'incite à investir en recherche le temps, les sommes et les efforts nécessaires au développement de nouvelles pratiques.

Pour remplir ces objectifs, le milieu universitaire, les employeurs et les praticiens doivent s'unir et participer à la recherche sur la pratique de la pharmacie. Selon moi, il reste beaucoup de chemin à faire.

Je crois que nous n'avons pas suffisamment de pharmaciens qui s'orientent vers la recherche sur la pratique de la pharmacie. Nous n'avons pas été en mesure d'atteindre la masse critique de chercheurs nécessaire pour démontrer l'effet substantiel qu'exerce l'évolution de notre pratique sur nos patients. Les facultés de pharmacie peuvent jouer un rôle important à ce sujet. Dans chaque province, combien les facultés ont-elles de postes dans les domaines du développement et de l'évaluation de la pratique de la pharmacie? Pas assez, je crois. Pour produire les données probantes sur les services prodigués par les pharmaciens, il faut avoir une formation clinique et une formation en recherche adéquates, une direction très engagée ainsi que les ressources et le temps voulus. Le milieu universitaire a un intérêt particulier dans ce domaine. Il peut d'ailleurs mettre en place les conditions nécessaires : à créer des réseaux de recherche permettant de rattacher les pharmaciens à leurs milieux de pratique, à ancrer la recherche dans le monde réel, à élargir les rôles des pharmaciens et, ultimement, à produire un effet positif sur les résultats thérapeutiques.

Les employeurs, notamment les organismes de soins de santé publics et privés, jouent un rôle dans l'adoption de nouvelles pratiques fondées sur des données probantes, mais aussi dans la production de nouvelles données probantes. Cela dit, du point de vue des organismes, la participation à la recherche fondée sur la pratique est souvent considérée comme un supplément à une description de poste, une activité intéressante qui parait bien dans un rapport annuel. Nous avons besoin que ces organismes offrent davantage de soutien administratif et de leadership en ce qui touche la promotion de la recherche fondée sur la pratique, de façon à valoriser la recherche et à en faire un élément central du rôle du pharmacien.

Peut-être les praticiens ne s'intéressent-ils pas suffisamment à participer à des recherches, ce que l'on considère souvent comme un travail du domaine universitaire et sans liens avec les 
services de soins aux patients. Mais des études par sondage ont montré que les pharmaciens d'hôpitaux et les pharmaciens communautaires du Canada voient la recherche d'un bon œil ${ }^{5,6}$. Lorsqu'on leur demandait s'ils seraient prêts à rejoindre un réseau de recherche, les répondants ont presque tous dit " oui " à cause des avantages potentiels qu'ils peuvent en tirer ${ }^{5,6}$. Parmi ces avantages, on compte : l'accès direct à des programmes de formation continue, l'occasion de participer à l'élaboration et à l'évaluation d'outils cliniques, l'accès à différents outils lorsqu'un projet de recherche est terminé, les connaissances acquises à la faveur des résultats de l'étude et, finalement, les avantages pour les patients qui sont sûrement les plus considérables. Par contre, ces mêmes études ont relevé que le manque de temps réservé à la recherche représente l'obstacle le plus important et le plus fréquent à la participation des répondants aux projets de recherche $e^{5-7}$, et ce, peu importe leur intérêt à l'égard de la recherche. Même si l'insuffisance de temps alloué semble constituer une réelle contrainte (selon la quantité de données qu'il est nécessaire de recueillir pour un projet de recherche donné), Cvijovic et collab. ${ }^{7}$, à l'aide d'entrevues semi-structurées, ont identifié le manque de temps comme un obstacle socialement acceptable masquant les peurs associées aux défis que présente le changement des habitudes de travail.

Les praticiens doivent surmonter leur réticence à modifier leur comportement et leurs habitudes de travail. Ils pourront ainsi être en mesure d'essayer et d'adopter les services éprouvés qui améliorent les résultats thérapeutiques. De même, les employeurs doivent considérer la recherche comme un élément central de la description de travail d'un pharmacien et le milieu universitaire doit mieux faire connaître les avantages de la recherche sur la pratique. Si nous devons réussir à fournir de nouveaux services fondés sur des données probantes et à observer un changement dans notre culture, nous devons amener nos étudiants et nos collègues à participer à cette activité d'érudition. Ils pourront donc constater les avantages qu'ils peuvent tirer de la recherche pour eux-mêmes, pour leur pratique, mais surtout pour leurs patients ${ }^{8}$. Si l'on reconnaît que les services de pharmacie en place sont le produit de recherches, alors nous disposons d'un argument simple, mais de taille pour l'adoption de cette culture : les recherches d'aujourd'hui ont le potentiel de produire les services cliniques de demain. Personnellement, je crois que le leadership est essentiel!

[Traduction par l'éditeur]

References

1. Tsuyuki RT, Schindel TJ. Changing pharmacy practice: the leadership challenge. Can Pharm J. 2008;141(3):174-80.

2. Awaisu A, Alsalimy N. Pharmacists' involvement in and attitudes toward pharmacy practice research: a systematic review of the literature. Res Social Adm Pharm. 2015;11(6):725-48.

3. Ernst AA, Weiss SJ, Sullivan A 4th, Sarangarm D, Rankin S, Fees M, et al. On-site pharmacists in the ED improve medical errors. Am J Emerg Med. 2012;30(5):717-25.

4. Tsuyuki RT, Rosenthal M, Pearson GJ. A randomized trial of a communitybased approach to dyslipidemia management: pharmacist prescribing to achieve cholesterol targets (RxACT study). Can Pharm J. 2016;149(5):283-92.

5. Perreault MM, Thiboutot Z, Burry LD, Rose L, Kanji S, LeBlanc JM, et al. Canadian survey of critical care pharmacists' views and involvement in clinical research. Ann Pharmacother. 2012;46(9):1167-73.

6. Hébert J, Laliberté MC, Berbiche D, Martin E, Lalonde L. The willingness of community pharmacists to participate in a practice-based research network. Can Pharm J. 2013;146(1):47-54.

7. Cvijovic K, Boon H, Jaeger W, Vohra S. Pharmacists' participation in research: a case of trying to find the time. Int J Pharm Pract. 2010;18(6):377-83.

8. Deal EN, Stranges PM, Maxwell WD, Bacci J, Ashjian EJ, DeRemer DL, et al. The importance of research and scholarly activity in pharmacy training. Pharmacotherapy. 2016;36(12):e200-5.

Marc M. Perreault, B. Pharm., M. Sc., Pharm. D., est pharmacien en soins critiques à l'Hôpital général de Montréal, Montréal, Québec. II est aussi rédacteur adjoint du Journal canadien de la pharmacie hospitalière.

Intérêts concurrents : Aucun déclaré.

Address correspondence to:

Dr Marc Perreault

Service de pharmacie

Hôpital général de Montréal

1650, avenue Cedar, bureau C1-200

Montréal (Québec) H3G 1A4

Courriel : marc.perreault@umontreal.ca 